ENCYCLOPEDDIE Encyclopédie berbère

BERBERE

$26 \mid 2004$

26 | Judaïsme - Kabylie

\title{
Kabylie : Chrétiens (de)
}

\author{
K. Direche-Slimani
}

\section{OpenEdition}

Journals

Édition électronique

URL : http://journals.openedition.org/encyclopedieberbere/1430

DOI : 10.4000/encyclopedieberbere. 1430

ISSN : 2262-7197

\section{Éditeur}

Peeters Publishers

\section{Édition imprimée}

Date de publication : 1 mai 2004

Pagination : 4050-4055

ISBN : 2-7449-0452-X

ISSN : 1015-7344

\section{Référence électronique}

K. Direche-Slimani, «Kabylie : Chrétiens (de) », Encyclopédie berbère [En ligne], 26 | 2004, document K14, mis en ligne le 01 juin 2011, consulté le 14 décembre 2020. URL : http://journals.openedition.org/ encyclopedieberbere/1430 ; DOI : https://doi.org/10.4000/encyclopedieberbere.1430

Ce document a été généré automatiquement le 14 décembre 2020.

(c) Tous droits réservés 


\title{
Kabylie : Chrétiens (de)
}

\author{
K. Direche-Slimani
}

1 Colonisation et évangélisation, conflictuelles mais indissociables, ont contribué à mettre en place, dans le dernier quart du XIx ${ }^{e}$ siècle, une communauté de chrétiens indigènes en Kabylie. Ce groupe d'hommes et de femmes, certes limité quantitativement (quelques milliers tout au plus dans les années 1920) s'est retrouvé, par les hasards de l'histoire, au cœur des paradoxes de la colonisation et d'une identité difficile à définir.

2 Le projet de conversion des Kabyles est à l'initiative de Charles de Lavigerie, évêque de Nancy nommé à l'archevêché d'Alger en 1867. Sa nomination à Alger lui laisse entrevoir la possibilité d'entreprendre un projet de conversion de grande ampleur; celle du continent africain tout entier. Selon lui, l'Algérie "n'est qu'une porte ouverte par la providence sur un continent barbare de deux cents millions d'âmes et que c'était là surtout qu'il fallait porter l'œuvre de l'apostolat catholique ». Dès sa nomination, il s'intéresse à la Kabylie qui retient toute son attention et mobilise une partie de son énergie. Sa position officielle ne lui permettant pas d'agir directement sur le terrain, il fonde la Société des Missionnaires d'Afrique, plus connue sous le nom de la Société des Pères Blancs. Ces derniers n'étaient pourtant pas les premiers religieux à s'installer en Kabylie. En effet, les missionnaires jésuites avaient déjà investi le terrain, à la fin des années 1840 , pour y implanter quelques postes mais sans réelle action concrète sur place. Ces Jésuites étaient présents pour encadrer les garnisons militaires et les quelques dizaines de civils qui s'étaient lancés dans l'aventure coloniale en Kabylie.

Lavigerie, dans son projet de christianiser la Kabylie, instrumentalisa toute l'imagerie coloniale qui alimenta abondamment le mythe kabyle de la deuxième moitié du XIx ${ }^{\mathrm{e}}$ siècle. Ce dernier accentuait particulièrement l'origine supposée européenne des Kabyles (germanique ou celte, voire romaine) et un certain nombre de traits communs avec les Européens qui aurait rendu leur assimilation possible. Sa vision caricaturale de l'histoire religieuse de l'Afrique du Nord selon laquelle tous les Berbères auraient adhéré à la religion chrétienne à l'époque romaine laissait supposer, selon lui, tout un fond de traditions et de pratiques chrétiennes qui ne demandaient qu'à resurgir. Des travaux statistiques, publiés dans les années 1860 et qui firent longtemps autorité, 
viennent renforcer cette thèse. On estimait, alors, que l'Algérie comptait 1200000 Berbères arabophones, 1000000 de Berbères berbérophones et 500000 Arabes. Les Berbères étant le nombre et les Arabes la minorité. L'exploitation de Lavigerie du mythe berbère notamment par son aspect fortement assimilationniste et les différentes expérimentations menées par les autorités coloniales ont fait de la Kabylie la région pilote de projets divers. Car elle réunissait, à elle seule, des critères spécifiques : densité de la population, sédentarité traditionnelle; mais aussi des changements qui annonçaient les mutations futures: les début de l'émigration, des institutions traditionnelles rendues caduques par l'administration coloniale, la confrontation avec l'économie de marché. Les tentatives d'évangélisation de Lavigerie s'inscrivent donc dans une politique coloniale plus globale. Région d'expérimentations politiques mais également région où les projections idéologiques étaient multiples.

La surenchère dans les qualités et vertus supposées des Kabyles prend avec Lavigerie et sa politique d'évangélisation toute sa dimension fantasmagorique.

5 Il n'est donc pas étonnant que le mythe kabyle, dans toutes ses incohérences et extravagances, ait servi de trame légitimante à ses projets. Il lui a même permis de prendre des libertés inconsidérées dans la lecture de l'histoire religieuse de l'Afrique du Nord. Libertés incongrues qu'on ne pourrait pas comprendre chez un personnage aussi érudit que le futur cardinal.

6 Les insurrections répétées des années 1850 et 1860 et une pacification tardive ont donc repoussé les projets d'une politique d'évangélisation en Kabylie. Il faudra, d'ailleurs, attendre l'instauration du régime civil (favorable à la colonisation de peuplement) et la marginalisation des militaires pour entreprendre quelques actions de colonisation rurale. La répression de 1871 et la législation foncière qui l'a suivie ont permis enfin à Lavigerie l'impulsion des postes missionnaires dans la Kabylie montagneuse. Il faut souligner que celle-ci se caractérise par une très faible présence de la population européenne (localisée dans deux pôles urbains: Fort-National et Michelet et représentée par les institutions majeures, l'école, l'hôpital et le tribunal) et une absence de colonisation rurale.

7 C'est donc dans une région traumatisée, appauvrie où l'organisation sociale et économique est particulièrement déséquilibrée (la tradition orale et poétique témoigne des souffrances des Kabyles qui s'interrogent sur ce monde nouveau, déstabilisant sinon effrayant qui se substitue à l'ordre ancien) que s'installent les missionnaires de la congrégation d'Afrique. Les Pères Blancs ont été les témoins particulièrement attentifs d'une époque de transformations et de bouleversements. En effet, le dernier quart du $\mathrm{XIX}^{\mathrm{e}}$ siècle est pour la Kabylie, un moment charnière de ruptures des équilibres et de mutations.

8 La question de la conversion se pose sur un espace géographique concernant cinq tribus (Ath-Ismail, Ath-Menguellat, Ath-Yenni, Ath-Douala, Ouadhias), au cœur du massif montagneux du Djurdjura. Ces cinq tribus sont les lieux d'implantation ancienne des missionnaires et ils correspondent également à des espaces de grande pauvreté et de marginalisation économiques associés à des situations fréquentes de dénuement. Trois voies de conversion ont été rendues possibles : par le baptême in articulo mortis (qui pouvait concerner les très jeunes enfants comme les adultes), par les soins médicaux et par l'école.

9 L'école étant soumise à une surveillance constante de la part des autorités administratives et des familles kabyles, c'est par l'action caritative et médicale que la 
très grande majorité des conversions s'effectue. Les petites écoles-internats du début de la mission ont permis de catéchuméner et de convertir quelques orphelins confiés aux missionnaires mais très rapidement la rumeur populaire, alimentée par certains administrateurs locaux, a freiné ce type d'action.

Par contre, la maladie et les soins médicaux, surtout quand ils sont accompagnés par une guérison, sont des moyens plus efficaces pour convertir. Dans ces conditions, la conversion devient, implicitement, un remerciement, une fidélité à celui ou celle qui a sauvé, presque une forme d'allégeance. Les missionnaires occupent, alors, grâce aux soins et aux remèdes, une place vide dans la société kabyle. Ils investissent un rôle social que presque personne ne joue, à l'exception de quelques rebouteuses et guérisseurs dont les conseils se révèlent bien trop souvent inefficaces.

11 Compte tenu de la difficulté du terrain kabyle et de la réserve méfiante de la population à l'égard des missionnaires, ces derniers ont préféré d'emblée s'intéresser au groupe social le plus défavorisé et le plus démuni car le plus accessible malgré les réserves que certains pères pouvaient émettre : "une chose qu'il faut éviter à tout prix, c'est d'avoir des catéchumènes que j'appellerais mercenaires, c'est-à-dire venant se faire instruire à cause des avantages temporels qu'ils espéraient trouver chez nous». Orphelins, démunis, veuves, marginaux sont pris ainsi en charge dans un cadre caritatif et scolaire.

Il s'agit, dans la très grande majorité des cas, de conversions de la misère qui ont concerné tout au plus quelques milliers d'individus. La conversion leur assurera un statut mal défini et des appellations variées : apostat, renégat ou m'tourni (littéralement celui qui a tourné le dos). Les conditions d'extrême pauvreté et de détresse qui sont souvent à l'origine de la conversion rendent la pratique de la religion catholique parfois problématique dans la mesure où elle ne correspond pas souvent à des cheminements spirituels ou à une adhésion totale au christianisme. Lorsque l'on restitue les trajectoires des familles de convertis, on constate qu'il existe des jeux de glissement, des effets de miroir, des ruptures qui pourraient sembler incompréhensibles à l'observateur extérieur. Bon nombre de convertis ont des parcours compliqués : il n'est par rare de rencontrer des individus nés musulmans, devenir chrétiens et exiger de mourir en musulmans en refusant l'extrême-onction. Le pourcentage d'échec de la conversion peut parfois être élevé. L'attribution des sacrements comme ceux du baptême ou du mariage prennent des sens différents à des moments clés d'une existence. Jouer avec les rituels de l'une ou l'autre religion, s'accommoder des pratiques sacralisées logiquement incompatibles et renforcer les adhérences à l'univers traditionnel musulman sont des choses fréquentes et communes dans le monde des convertis de la première et deuxième génération.

13 Les chiffres de baptêmes dont nous disposons sur le village cité ci-dessous et puisés dans les archives des Pères blancs permettent d'évaluer le nombre de convertis et surtout d'estimer le patient travail des Pères blancs sur plus de quatre-vingt ans.

\section{Village de Taourirt Abdallah-Ouadhias (Paroisse Notre- Dame des Sept Douleurs)}

14 Entre 1888 et 1970, on dénombre :

- 528 baptêmes (dont une proportion non négligeable d'enfants et adolescents), soit une 
moyenne de 6,3 baptêmes par an ;

- 104 mariages, soit une moyenne de 1,25 mariage par an.

Ce groupe de Kabyles chrétiens est marqué par un processus migratoire précoce. Dès les années 1920, et pour certaines au cours de la décennie précédente, les familles émigrent, dans un premier temps à Alger et dans les grandes villes algériennes puis en Tunisie; et dans un second temps (années 1950-1960) vers la France. Il semblerait que, dès les premières conversions, cette émigration ait été inévitable. Instruits, diplômés, encadrés par les missionnaires, les Kabyles chrétiens ont très tôt formé une élite sociale et professionnelle. D'où une ascension sociale spectaculaire dans un cadre aussi pauvre et rural que celui de la société kabyle, mais également une déconnexion tout aussi rapide avec le milieu d'origine. La conversion ayant été probablement le facteur déterminant à leur émergence dans la société kabyle (car la réussite a été facilitée par un cadre scolaire missionnaire performant) mais également facteur d'exil et de déracinement. Jean Amrouche, le Kabyle chrétien le plus célèbre sans doute, a remarquablement montré la douleur de cet exil et le malaise identitaire ressenti surtout par les deux premières générations.

\section{Les convertis : ni Français ni Musulmans}

L'existence des convertis va poser très tôt la question de leur statut et de leur citoyenneté. Si on constate des naturalisations précoces de certains Kabyles chrétiens et ce, dès les années 1880 , l'accès à la citoyenneté française suppose toujours des interrogations et des démarches difficiles.

17 La naturalisation, avec sa procédure pourtant compliquée, s'est souvent présentée comme la seule issue pour éviter toutes les difficultés qui se manifestaient lorsque les convertis avaient affaire à l'administration (enregistrement à l'état-civil, mariages, armée, justice). Mais elle était également encouragée par les missionnaires qui y voyaient la consécration exemplaire d'une assimilation réussie. Le converti qui se naturalise vit ainsi une double stigmatisation : celle alimentée par les colons qui pointe son statut d'ancien indigène et celle projetée par la mémoire populaire de l'apostat, du traître qui y voit l'exemple honteux du rejet du statut personnel et du reniement de ses racines. Cette dernière stigmatisation bénéficiera d'une surenchère lors des années post-indépendantes. Le néo-patriotisme algérien fondé sur la devise de Ben Badis, leader des 'Ulémas réformateurs : «l'Islam est ma religion, l'arabe ma langue et l'Algérie ma patrie » ne laissera aucune possibilité d'expression à d'autres affirmations identitaires.

\section{La guerre d'Algérie : engagements et identité hybride}

C'est la guerre d'Algérie qui allume les projecteurs sur le statut des convertis en exigeant d'eux des positions identitaires et nationales en faveur de la lutte indépendantiste.

Pour des individus que les hasards de la colonisation ont singularisés et qui n'étaient pas forcément outillés pour s'exprimer et se positionner, c'est certainement la période la plus difficile de leur histoire. Conscients, peut-être plus que d'autres - car au carrefour de toutes les stigmatisations - de la violence du système colonial, de ses injustices et de ses discriminations, ils réalisent très rapidement la complexité de leur 
statut et des prises de position qu'on attendait d'eux. Impliqués, déjà, dans les mouvements anti-coloniaux des années 1920 et 1930, ils combattaient pour une plus grande justice. Cette position intégrationniste recueillait les faveurs d'un grand nombre d'élus indigènes qui luttaient, par ailleurs, pour l'égalité des droits et d'un véritable statut pour les Algériens.

Il n'y a pas eu de représailles à l'encontre des convertis. Leur choix religieux, n'a paradoxalement alimenté ni haine, ni désirs de vengeance. La Congrégation des Missionnaires d'Afrique, ni la communauté des Kabyles chrétiens n'ont eu à subir, de la part, des maquisards puis de l'ALN des actes de violences. Leur destin n'a rien à voir avec ce que les Harkis auront à subir à l'indépendance. Leur extrême discrétion, l'engagement emblématique et la mort au combat de certains d'entre eux, l'absence d'organisation communautariste, les a préservés de la folie meurtrière qui s'est abattue sur l'Algérie pendant ces années. L'engagement de l'Eglise catholique algérienne en faveur du FLN et de l'indépendance, le charisme d'une personnalité comme celle de Monseigneur Duval, archevêque d'Alger ont certainement contribué à la protection de ces chrétiens incongrus. Il n'y a pas de témoignages de morts civiles comme on a pu en observer dans d'autres pays. Les convertis n'ont pas été livrés à la vindicte populaire mais leur présence va être progressivement liée au complexe du non-arabe et du nonmusulman dans une Algérie qui proclame orgueilleusement une identité exclusivement arabe et musulmane.

21 À leur manière, les convertis participent également à la tradition migratoire kabyle. Si l'écrasante majorité des convertis se retrouve en France, après les années qui ont suivi l'indépendance, ils insistent pour préciser qu'ils ne sont pas partis, dans l'urgence et la violence, comme les Pieds-noirs et les Harkis. D'ailleurs un grand nombre d'entre eux avaient déjà des membres de leur famille installés en France. Il n'y a pas eu d'exode et les départs se sont faits progressivement. Pourtant, les tentatives de rester en Algérie ont été nombreuses; certaines ont réussi brillamment. Les Kabyles chrétiens ont parfois été courtisés par le pouvoir algérien. Dans une Algérie, ruinée par huit années de guerre violente, où $94 \%$ des hommes et $96 \%$ des femmes étaient analphabètes, ils représentaient un capital précieux de compétences et de qualités professionnelles mais aussi, paradoxalement, une réponse à une sorte de pluralisme culturel voulu par l'Etat algérien en réaction aux départs les Pieds-noirs vers la France.

Ce n'est pas l'indépendance qui leur a fait quitter l'Algérie mais bien le dogmatisme de l'Etat algérien qui se radicalise après 1965 . Une radicalisation dans l'arabo-islamisme, dans le verrouillage de la société civile et dans les pratiques policières de l'appareil d'Etat. Leur berbérité et leur christianisme les ont doublements stigmatisés dans une définition de l'identité nationale qui ne leur accordait aucune reconnaissance. D'ailleurs l'évocation des tracasseries administratives, dans un contexte de politique d'arabisation acharnée, s'inscrit dans ces années post-indépendantes. Ne parlant pas l'arabe, ayant des prénoms chrétiens, francophones (mais comme l'écrasante majorité de l'élite algérienne de l'époque), ils sont progressivement singularisés et renvoyés dans des positions de silence et de gêne. La politique religieuse agressive de l'Etat algérien qui prit certaines mesures de rétorsion ne fera que renforcer le malaise. La gêne, le sentiment de ne pas être à sa place et d'être peut-être surveillés, l'incommodité de leur situation poussent progressivement les convertis à partir. Leur identité n'est décidément pas simplifiable. La France les accueille plutôt correctement et leur a très souvent proposé des situations, non pas équivalentes à celles qu'ils occupaient en 
Algérie, mais globalement assez satisfaisantes. Là encore, il faudrait s'interroger sur les perceptions de la France à leur égard. Les a-t-elle considérés comme un groupe à préserver, à protéger ? Leur statut de chrétiens et de Kabyles en a-t-il fait une catégorie privilégiée? Leur intégration dans la bourgeoisie moyenne française, par leurs fonctions dans l'administration et l'armée, leur a évité d'être malmenés économiquement et socialement. Mais si leur discrétion et surtout leur faible nombre ont permis une réinsertion professionnelle assez réussie, le sentiment d'exil demeure très fort. Aujourd'hui, leurs descendants cherchent à comprendre les paradoxes inhérents à la rencontre historique (colonisation et évangélisation) qui a produit leur identité et à défendre une certaine idée de la pluralité culturelle.

\section{BIBLIOGRAPHIE}

AMROUCHE F., Histoire de ma vie, Editions Maspero, 1968, (réédité à La Découverte, 1991).

DIRECHE-SLIMANI K., « Père Blancs de Kabylie », Hommes et femmes de Kabylie, I, (Dictionnaire biographique de la Kabylie), Aix-en-Provence, Édisud, 1981.

DIRECHE-SLIMANI K, Chrétiens de Kabylie (1873-1954). Une action missionnaire dans l'Algérie coloniale, Editions Bouchêne, Paris, 2004.

EMERIT M., « La lutte entre les généraux et les prêtres aux débuts de l'Algérie Française », Revue Africaine, 1960.

EMERIT M., « Le problème de la conversion des musulmans d'Algérie sous le Second Empire. Le conflit entre Mac Mahon et Lavigerie », Revue Historique, 1960. LAVIGERIE C. Mgr, Missionnaires d'Afrique, recueil de textes et de discours, Editions SOS, Paris, 1980.

RENAULT F.s, Le cardinal Lavigerie 1825-1892. L'Eglise, l'Afrique et la France, Fayard, Paris, 1992.

\section{INDEX}

Mots-clés : Christianisme, Kabylie, Religion 